\title{
Quality of Service Aware Reverse Pathad-Hoc On-Demand Distance Vector Protocol
}

\author{
${ }^{1}$ Santhiya, K.G. and ${ }^{2}$ N. Arumugam \\ ${ }^{1}$ Department of Computer Science, \\ Mother Teresa Women University, Kodaikkanal, Tamilnadu, India \\ ${ }^{2}$ Department of Information Technology, \\ MNM Jain Engineering College, Anna University, Chennai, Tamilnadu, India
}

Received 2012-04-20, Revised 2012-09-16; Accepted 2012-09-20

\begin{abstract}
Mobile Ad-hoc Network (MANET) is aninfra structureless network that does not require any built-in infra structure and central control. Due to very fast mobility of nodes, there will be drastic changes in the network topology. So an adaptive routing protocol is needed for effective and reliable routing in MANET. Ad-hoc On-demand Distance Vector (AODV) routing protocol is the efficient and prominent routing protocol which may satisfy the above said issue. While making routing process in traditional AODV,onlyone route information will be sent as reply in the reverse path. This reply from the destination may be lost due to fast mobility of modes which in turn leads to the retransmission of route request packets to establish routes. The main cause of this problem is increased communication delay and reduction of several QoS metrics. The main goal of the new Novel QoS aware reliable routing algorithm is to send multiple route replies and establishes reliable data transmission between source and destination. The new NQARR-AODV protocol establishes paths on-request using a reliable reverse pathestablishment method. During pathestablishmentphase, the source node first sends the route requests through all available paths to the destination. The destination node,upon receiving the path requests,inturn rebroadcasts the reverse path requestsas like sender node has done. Upon receiving the multiple route replies from the destination, a path with high reliability is choosen by the source node using the three parameters, the MAC overhead, the Eminence of Link, node residual energy. NQARR-AODV path establishment method works well and requires only minimum steps. It also provides high energy efficiency and load balancing thus prolongs the network life time and makes up high reliability communications. The experimental values prove that the NQARR-AODV algorithmis more reliable and stable and outperforms AODV by reducing energy consumption, overhead and delay. Also NQARR-AODV gains bestratio of packets delivered than that of normal AODV. In our proposed NQARR-AODV protocol, the source node can find QoS aware reliable route to the destination.
\end{abstract}

Keywords: Mobile Ad Hoc Networks (MANETs), Ad-hoc On-Demand Distance Vector (AODV), Routing Protocol, Reverse AODV, Quality of Service (QoS)

\section{INTRODUCTION}

A MANET is a multi-hop wireless network which doesnot require any basic infra structure. Due to very high mobility of nodes, the topology of the network changes drastically which make the routing processmore difficult and crucial (Yen et al., 2010; Saleem et al., 2010a). Many routing protocols are available for
MANETs. There are two types of routing algorithms. One is Proactive routing algorithm (table driven) and reactive routing algorithm (On-demand) (Abusalah et al., 2008; Zhang and Anpalagan, 2010).

In proactive routing algorithm,the routing information needs to be exchanged between nodes at regular intervalsand the routes are calculated whether the routes are needed or not. The main disadvantage of this

Corresponding Author: Santhiya, K.G., Department of Computer Science, Mother Teresa Women University, Kodaikkanal, Tamilnadu, India 
method is the wastage of network resources (Verma et al., 2010; Hou et al., 2012). In on-demand routing algorithms, the nodes need not exchange routing information between them. The nodes find paths only when they are needed for data transfer.

The very fast mobility of nodes leads to the unstable links between nodes. The unstable links may cause the loss of data packets as route reply control messages. The path establishment process has to be carried out several times by the source node (Papageorgiou et al., 2008; Farooq, 2009). The loss of route reply messages are not handled well by the available on-demand routing algorithms which leads to the reduction of network performance (Huang et al., 2008; Hou et al., 2012).

While establishing only shortest route between source and destination, the poor utilization of network resources is unavoidable. In order to maximize the usage of network usage, routing of network must find routes with high stability and sufficient energy level (Chen et al., 2008; Madan et al., 2009; Saleem et al., 2010b).

The main aim of the proposed protocol Novel QoS aware reliable routing protocol is to send multiple route replies and establishes data transmission between source and destination and achieves high reliability, stability, low latency and outperforms AODV by reducing energy consumption, overhead and delay (Lim et al., 2009; Zhang et al., 2009).

Our proposed NQARR routing algorithm works well and requires only minimum steps for path establishment.In order to select a reliable route, proposed protocol uses three parameters, the MAC overhead, the Eminence of Link, node residual energy. It also provides high energy efficiency and load balancing thus prolongs the network life time and makes up high reliability communications.

\section{MATERIALS AND METHODS}

\subsection{NQARR-AODV}

\subsection{Proposed Routing Protocol}

NQARR-AODV is an on-demand multipath QoS Aware Reliable Reverse Path routing protocol for finding routes in MANET.

The new NQARR-AODV protocol establishes paths on-request using a reliable reverse pathestablishment method. During pathestablishment phase, the source node first sends the route requests through all available paths to the destination. The destination node upon receiving the path requests,inturn rebroadcasts the reverse path requestsas like sender node has done. Upon receiving the multiple route replies from the destination, a path with high reliability is choosen by the source node using the three parameters, the MAC overhead, the Eminence of Link, node residual energy. NQARRAODV path establishment method works well and requires only minimum steps. It also provides high energy efficiency and load balancing thus prolongs the network life time and makes up high reliability communications.After the link establishment, the source node will issue command to put the neighbouring nodes in sleep state; hence the node remaining energy will expand for long period.

\subsection{MAC Contention and Overhead Estimation}

The parameters Requestion-for-Sending (RS), Clearfor-Sending (CS) and Acknowledge-for-Data (ACKD), Space Between Frames (SBF) which is the time gap between the transmission of two consecutive packets are used to calculate the Channel workload:

$\mathrm{CW}=\mathrm{tRS}+\mathrm{tCS}+\mathrm{tSBF}$

The MAC contention value calculated using Eq. 1 is used to calculate the MAC overhead $\mathrm{MOH}$ as follows:

$\mathrm{MOH}=\mathrm{CW}+\mathrm{tac}$

where, tac is the amount of time consumed by the contention of access.

\subsection{Estimation of Link-Eminence}

The link eminence is computed at the physical layer and will be used and accessed at the top layers for routing. The estimation of link eminence for the neighbouring nodes is discussed here. In the physical layer the measured link eminence value is reassigned towards the MAC layer. This assigned value is kept in the neighbouring nodes and is used for optimization when more nodes are in the sending node's transmitting capacity. The link eminence optimizes the routing decisions and improves the capability of the networks.

The IEEE 802.11 is fairly reliable MAC protocol. The link eminence has reached every exposed node; it assumes the fixed utmost transmission power. Each sender node that transmits the Requestion-for-Sending (RS) packet, attaches its transmissions power details. The receiving node estimates the link eminence received for the freespace propagation model while receiving the RTS packet:

$\mathrm{LE}=\left(\lambda / 3.14 *\left[\mathrm{D}_{\mathrm{sr}]}\right) * \mathrm{~T}_{\mathrm{g}} * \mathrm{~T}_{\mathrm{r}}\right.$

where, $\lambda$ is the wavelength of the transmission carrier, $D_{\text {sr }}$ gives the informaion about how far the source and receiver are from each other, $\mathrm{T}_{\mathrm{g}}$ and $\mathrm{T}_{\mathrm{r}}$ are variables that depicts the antenna's unity gain. 


\subsection{Estimation of Residual Energy Level}

The energy for one packet delivery relative to the node distance is given as:

$\mathrm{PTE}=\mathrm{c} * \mathrm{dst}^{\alpha}$

where, $\mathrm{c}$ is the constant value, dst depicts that how far the neighbouring nodesare andthe parameter ${ }^{\alpha}$ depends on the physical location. Only less amount of energy is needed for the nearer nodes.

The PTE value calculated using the Eq. 4 is used to calculate the packet transmitting energy as follows Eq. 5:

$\mathrm{E}=\frac{\mathrm{DPS} * \mathrm{PTE}}{\mathrm{BW}}$

where, DPS is the size of each data packet, PTE is the packet transmitting energy and $\mathrm{BW}$ is the bandwidth of the wireless link.

In each and every node, the Required Energy for Transmission is calculated using the following formula:

$\mathrm{RTE}=$ pkts $*(\mathrm{PTE}-\mathrm{PPE})$

here, pkts is the amount of data packets. The Energy needed for Processing Packet (PPE) which is lessar than the PTE. Node Residual Energy (NRE) is calculated using the following formula:

$\mathrm{NRE}=(\mathrm{IE}-\mathrm{TER})$

where, NRE is the Node Residual Energy, IE is the Initial Energy and TER is the total energy required.

\subsection{Route Discovery}

The data transmission is initiated by the sender by broadcasting path request packets to all of the neighbouring nodes towards the destination node.

The ID for broadcasting each route request message is incremented. The ID for broadcastingis used to uniquely identify the RREQ packet (Wang and Lee, 2009; Yen et al., 2010). The path request control packet is broadcasted by the source node of the neighbouring nodes. This process is repeated by all the neighbouring nodes. Duplicate copies of the path request may reach the intermediate nodes. In this case, the nodes checks for redundancy and drops the redundant packets, if any.

This method is followed for the reverse path request processing also.

Upon the receipt of first route request message, the destination it first appends the RREQ packet information to its own routing table and after that the path request is made in reverse and broadcast to all of its neighbours as done previously.
When broadcasted R-RREQ message arrives to intermediate node, it will check for redundancy. If it already received the same message, the message is dropped, otherwise forwards to next nodes.

Upon receiving multiple Reverse Request packets from the destination, the initiator node finds reliable path with the three estimated parameters using the Eq. 2, 3, 6 and 7. They are MAC Overhead, Link Eminence and Node Residual Energy. After estimating the aforesaid parameters the following main parameter for route selection will be carried out using:

$\mathrm{W}=\min \left(\int_{\mathrm{i}=1}^{\mathrm{i}=\mathrm{nn}} \mathrm{LE} *\left(\frac{1}{\mathrm{MOH}}\right)+\mathrm{RTE}\right)$

The route with the minimum weight value which is calculated using the Eq. 8 is selected as best route and starts data transmission.

\section{RESULTS}

Extensive simulation experiments were conducted using the simulator Qualnet. The following list shows the simulation environment.

Simulation parameters:

Terrain Dimension:

$1500 * 1500$

Number of nodes:

50

Mobility model:

Propagation model:

MAC protocol:

Simulation time:

Antenna type:

Random way point

Two-ray Rayleigh fading

IEEE 802.11 DCF

Transmission range $(\mathrm{m}): \quad 200$

Node speed $(\mathrm{m} / \mathrm{s})$ :

Traffic type:

$300 \mathrm{sec}$

Omni directional

Traffic rate:

$0,5,10,15,20$

CBR

Initial Energy:

10packets/s

0.5 Joules

\section{DISCUSSION}

\subsection{Performance Metrics}

The performance of NQARR is evaluated against AODV protocol using the following metrics:

- Ratio of Packets Delivred: The ratio between the total packets produced at the source node and the packets delivered to the destination

- End-to-End Delay: The gap time of sending and receiving packets with the inclusion of queue waiting, packet processing time

- Total Energy Consumed: The average amount of energy consumed by every node 
Santhiya, K.G. and N. Arumugam / Journal of Computer Science 8 (11) (2012) 1854-1858

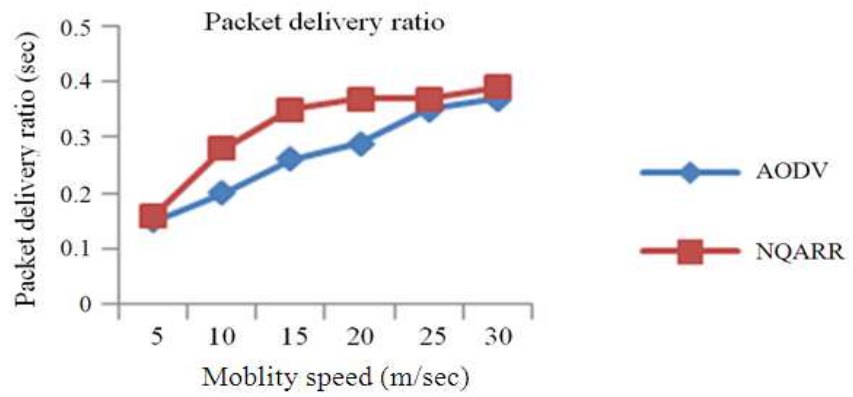

Fig. 1. Ratio of PacketsdeliveredVs mobility speed

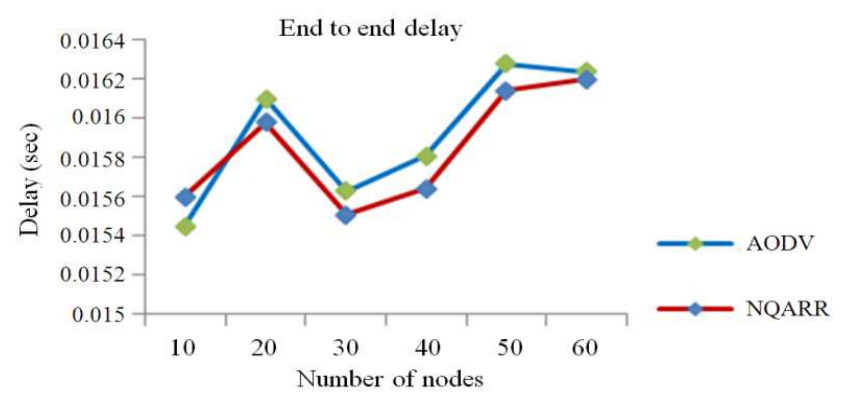

Fig. 2. End to end delay Vs number of nodes

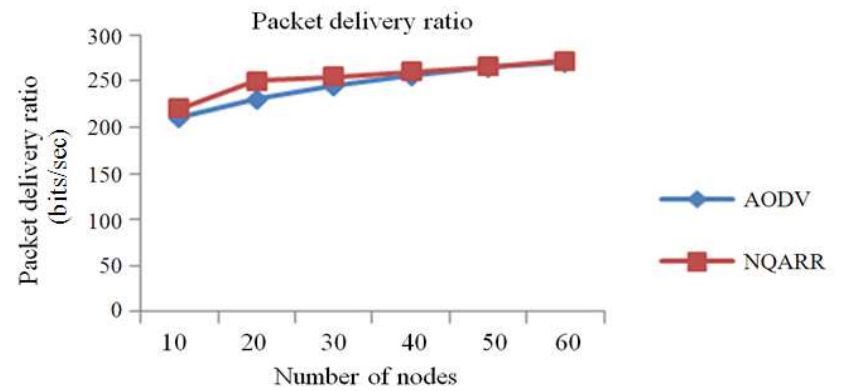

Fig. 3. Ratio of Packets DeliveredVsNumber of Nodes

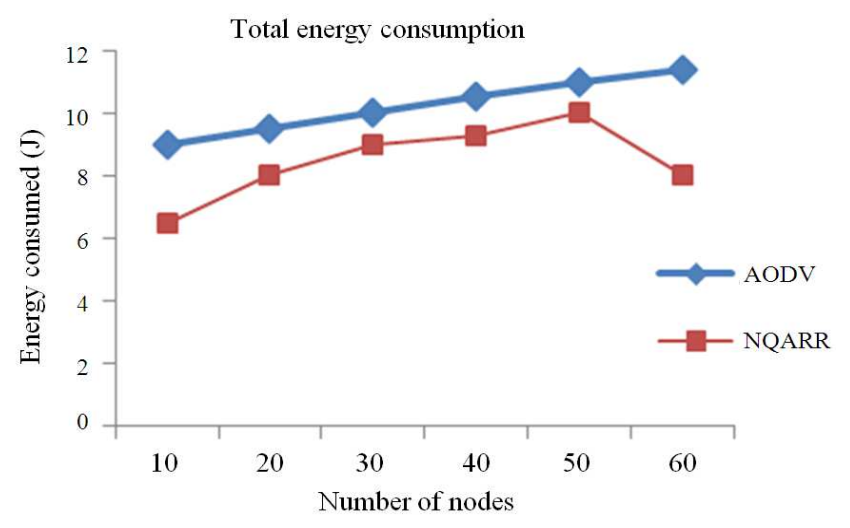

Fig. 4. Number of nodes VsTotal Consumed Energy 
The results in Fig. 1 show that the Ratio of Packets Deliveredbythe new NQARR algorithm is higher than the traditional AODV in varying mobility speed.

From Fig. 2 it is proven that the end to end delay of AODV is higher than the proposed protocol.

Figure 3 shows that the acheived packet delivery ratio of proposed NQARR protocol is higher than the traditional AODV.

Regarding Total energy consumption of nodes, Fig. 4 proves that only lesser energy is consumed while using the proposed NQARR protocol in contrast with the AODV.

\section{CONCLUSION}

A new Novel QoS Aware reliable reverse Ad-hoc On-Demand Distance Vector routing protocol for MANET has been proposed in this study. The route reply control message in on-demand routing protocol must be delivred correctly. The improper or loss of route reply packets leads to the worse case scenerio in the network. In this case, the path establishment process has to be initiated again by the source node. The new algorithm attempts reverse route requests and finds reliable path in minimal steps. In order to select a reliable path, proposed protocol uses three parameters, the MAC overhead, the Eminence of Link, node residual energy. It also provides high energy efficiency and load balancing thus prolongs the network life time and makes up high reliability communications. The experimantal values prove that the new scheme works well than AODV in discovering and maintaining routes and outperforms AODV in several qualities such as the ratio of packets delivered, end to end delay andtotal consumed energy.

\section{REFERENCES}

Abusalah, L., A. Khokhar and M. Guizani, 2008. A survey of secure mobile ad hoc routing protocols. IEEE Commun. Surv. Tutorials, 10: 78-93. DOI: 10.1109/SURV.2008.080407

Chen, M., T. Kwon, S. Mao, Y. Yuan and V.C.M. Leung, 2008. Reliable and energy-efficient routing protocol in dense wireless sensor networks. Int. J. Sensor Netw. DOI: 10.1504/IJSNET.2008.019256

Farooq, M., 2009. Bee-Inspired Protocol Engineering: From Nature to Networks. 1st Edn., Springer, Berlin, ISBN-10: 3540859535, pp: 306.

Huang, J.H., L.C. Wang and C.J. Chang, 2008. QoS provisioning in a scalable wireless mesh network for intelligent transportation systems. IEEE Trans. Veh. Technol., 57: 3121-3135. DOI: 10.1109/TVT.2008.918701
Hou, R., K.S. Lui, F. Baker and J. Li, 2012. Hop-by-hop routing in wireless mesh networks with bandwidth guarantees. IEEE Trans. Mobile Comput., 11: 264277. DOI: 10.1109/TMC.2011.25

Lim, S., C. Yu and C.R. Das, 2009. Random cast: An energy-efficient communication scheme for mobile ad hoc networks. IEEE Trans. Mobile Comput., 8: 1039-1051. DOI: 10.1109/TMC.2008.178

Madan, R., N.B. Mehta, A.F. Molisch and J. Zhang, 2009. Energy-efficient decentralized cooperative routing in wireless networks. IEEE Trans. Automatic Control, 54: 512-527. DOI: 10.1109/TAC.2009.2012979

Papageorgiou, C.A., P.C. Kokkinos and E.A. Varvarigos, 2008. Implementing distributed multicost routing in mobile ad hoc networks using DSR. Proceedings of the 6th ACM International Symposium on Mobility Management and Wireless Access, (MobiWac' 08), ACM Press, USA., pp: 35-42. DOI: 10.1145/1454659.1454666

Saleem, M., I. Ullah, S.A. Khayam and M. Farooq, 2010a. On the reliability of ad hoc routing protocols for lossand-delay sensitive applications. Ad Hoc Netw., 9: 285-299. DOI: 10.1016/j.adhoc.2010.07.012

Saleem, M., S.A. Khayam and M. Farooq, 2010b. On performance modeling of ad hoc routing protocols. EURASIP J. Wireless Commun. Network. DOI: 10.1155/2010/373759

Verma, P.K., T. Gupta, N. Rakesh and N. Nitin, 2010. A mobile ad-hoc routing algorithm with comparative study of earlier proposed algorithms. Int. J. Commun. Netw. Syst. Sci., 3: 289-293. DOI: 10.4236/ijens.2010.33037

Wang, N.C. and C.Y. Lee, 2009. A reliable QoS aware routing protocol with slot assignment for mobile ad hoc networks. J. Netw. Comput. Appl., 32: 11531166. DOI: 10.1016/j.jnca.2009.06.001

Yen, Y.S., H.C. Chang, R.S. Chang and H.C. Chao, 2010. Routing with adaptive path and limited flooding for mobile ad hoc networks. Comput. Elect. Eng., 36: 280290. DOI: 10.1016/j.compeleceng.2009.03.002

Zhang, L., Q. Wu and A. Solanas, 2009. A scalable robust authentication protocol for secure vehicular communications. IEEE Trans. Vehic. Technol. DOI: 10.1109/TVT.2009.2038222

Zhang, N. and A. Anpalagan, 2010. Comparative review of QoS-aware on-demand routing in ad hoc wireless networks. Wireless Sensor Network, 2: 274-284. DOI: 10.4236/wsn.2010.24038 